Short communication

\title{
Comparative study of hatching estimation methods of Rhipicephalus (Boophilus) microplus eggs
}

\author{
Amanda Figueiredo ${ }^{\mathrm{a}, *}$, Isabela Cabeça Agnolon ${ }^{\mathrm{b}}$, Louyse Gabrielli Lopes ${ }^{\mathrm{b}}$, Rodrigo Giglioti ${ }^{\mathrm{a}}$, \\ Ana Carolina de Souza Chagas ${ }^{c}$ \\ ${ }^{a}$ Faculdade de Ciências Agrárias e Veterinárias, Universidade Estadual Paulista Júlio de Mesquita Filho, Jaboticabal, São Paulo, Brazil \\ ${ }^{\mathrm{b}}$ Centro Universitário Central Paulista, São Carlos, São Paulo, Brazil \\ ${ }^{\mathrm{c}}$ Embrapa Pecuária Sudeste, São Carlos, São Paulo, Brazil
}

\section{A R T I C L E IN F O}

\section{Keywords:}

Cattle tick

Methods of larvae estimation

Visual estimation

Quantification by sampling

Adult immersion test (AIT)

\begin{abstract}
A B S T R A C T
Reproductive parameters of Rhipicephalus (Boophilus) microplus are often evaluated. They are good indicators of resistance to commercial acaricides and of plant extracts' efficacy. The objective of this study was to compare the techniques: visual estimation and quantification by sampling used in the Adult Immersion Test (AIT) to calculate the hatching rate of eggs. Engorged females collected from cattle were subjected to the AIT with plant extracts and kept in an incubator for oviposition. The egg hatching was evaluated in 210 syringes by visual estimation (\%). Then, eggs and larvae were counted into samples of 100 individuals, in three repetitions by stereo microscope. Significant differences were found between the two tests $(p \leq 0.05)$. The egg hatching average of visual estimation was higher than the quantification by sampling, 56.8-48.0, respectively (correlation $=0.85$ ). We found that the visual assessment leads to a higher estimate of larvae in relation to eggs, because the infertile eggs can be concealed in the center of the syringe. In quantification by sampling, no statistical differences $(p=0.99)$ were observed in the pairwise counts between the three samples $(48.1 \pm 26.6 \%, 47.8 \pm 26.9 \%$, $48.1 \pm 26.5 \%$ ) (correlation of repetitions $=0.96$ ). This suggests that counting one sample is sufficient and the result should not differ much, regardless of the evaluator. Regarding the cutoff point of tick resistance status (95\%), both methods are reliable. This study contributes to improvement of the AIT and can stimulate researchers to choose more accurate techniques for the assessment of egg hatching.
\end{abstract}

\section{Introduction}

Rhipicephalus (Boophilus) microplus is an ectoparasite considered of extreme importance in world livestock breeding because of the damage it causes. In addition to compromising the well-being of animals due to blood spoliation and disease transmission, it also causes reduction of productive performance in tropical and subtropical regions (Raynal et al., 2013). As a result, economic losses are high, aggravated by the transmission of agents of cattle tick fever. In Brazil, the losses are estimated at around $\$ 3.24$ billion per year, from lower meat and milk production, hide damage and costs for purchase and application of acaricides (Grisi et al., 2014).

The frequent and indiscriminate use of acaricides increases the presence of residues, which invariably can harm the environment as well as animal and human health (Schwarzenbach et al., 2010). The selection for resistance to major classes of acaricides widely used has been extensively reported (Raynal et al., 2013). Recently, the results of
Reck et al. (2014) demonstrated the first case of fluazuron resistance in $R$. microplus and the first tick population resistant to six classes of acaricides in Brazil.

The standardization of tests for the diagnosis of resistance against chemotherapeutic agents in biological organisms is a key requirement for the development of an applicable diagnostic tool allowing directly comparable data to be produced in different laboratories. Moreover, the ability to reliably detect parasite resistance is a crucial part of resistance management (von Samson-Himmelstjerna et al., 2009). According to Gaur et al. (2016), the Food and Agriculture Organization (FAO) and World Association for the Advancement of Veterinary Parasitology (WAAVP) have recommended and provided standardized protocols for bioassays to evaluate resistance, namely the Larval Packet Test (LPT) and the Adult Immersion Test (AIT), the latter originally developed by Drummond et al. (1973). The Larval Immersion Test (LIT) has also proved better at discriminating between resistant and susceptible strains to macrocyclic lactones (Klafke et al., 2012). For decades, the

\footnotetext{
* Corresponding author at: Embrapa Pecuária Sudeste, Rod. Washington Luiz, km 234, Caixa Postal 339, São Carlos 13560-970, São Paulo, Brazil.

E-mail address: amanda.figueiredo@colaborador.embrapa.br (A. Figueiredo).
} 
AIT has also been used as a tool for screening new active molecules present in plant extracts, including fractions, isolated and synthesized substances, with potential for tick control (Habeeb, 2010).

In the AIT, engorged female ticks are immersed in technical or commercial grade acaricides and the assay is interpreted based on the rate of oviposition between the two groups (treated and control). In this test, the percentage of egg hatching is estimated visually and applied in a formula to calculate the estimated reproduction (ER) of the engorged females, and consequently the efficacy of the substance in question. However, Amaral (1993) stated that the visual assessment of egg hatching is subjective and can vary when carried out by different technicians. Thus, since hatching percentage is a parameter routinely used in the AIT, the purpose of this study was to compare the techniques of visual estimation and quantification by sampling for calculation of the hatching rate of eggs laid by engorged females incubated in the laboratory.

\section{Material and methods}

\subsection{Adult immersion test (AIT)}

To measureegg hatching rates, engorged females of Rhipicephalus (Boophilus) microplus were previously submitted to trials with essential oils from plants in different concentrations and controls (Chagas et al., 2016). So, in the present study we used the egg hatching rates of ticks exposed to them. Briefly, engorged females $(\geq 4.5 \mathrm{~mm}$ ) were collected from naturally infested cattle at the experimental farm of Embrapa Pecuária Sudeste (CPPSE). Engorged females were divided into three groups of 10, weighed to establish uniformity among the different experimental groups, and placed in disposable cups to perform the immersion test with the essential oil samples. For each oil, seven dilutions were tested in triplicate: $25,12.5,6.25,3.12,1.56,0.78$ and $0.39 \mathrm{mg} /$ mL. Distilled water and $2 \%$ Tween 80 were used as control treatments, also in triplicate. The females were immersed in $5 \mathrm{~mL}$ of solutions for $5 \mathrm{~min}$, dried and placed in Petri dishes in an incubator $\left(27 \pm 1{ }^{\circ} \mathrm{C}\right.$ and $\mathrm{RH} \geq 80 \%$ ) for oviposition. After 18 days, eggs were weighed and transferred to adapted transparent plastic syringes $(10 \mathrm{~mL})$, identified, and sealed with cotton. They were then placed back in the incubator under the same conditions for egg hatching (Drummond et al., 1973). After 15 days, the hatching rate was evaluated in 210 syringes. The visual reading was performed by a single trained technician, while the quantification by sampling was done by three technicians.

\subsection{Visual estimation}

The egg hatching rate of each syringe was first evaluated by visual estimation, given as a percentage, by observing the proportion of larvae in relation to eggs not hatched. This was done by just one visual observation according to the technique described by Drummond et al. (1973). The trained technician had no knowledge about the treatment of the syringes, to avoid biased estimation.

\subsection{Quantification by sampling}

After the visual reading, the syringes containing larvae were kept in an oven, with controlled temperature $\left(50^{\circ} \mathrm{C}\right.$, Solab SL-100) for $24 \mathrm{~h}$ to kill them and allow the counting. The contents of each syringe (hatched larvae, egg shells and infertile eggs) were homogenized in a disposable cup using a spatula. Randomly collected samples were distributed in disposable Petri dishes $(90 \times 15 \mathrm{~cm}$ with three partitions) scratched at the base to facilitate the counting (Fig. 1). In this way, there were three counts (eggs or larvae) into samples of 100 individuals, with the aid of a cell counter and a stereoscopic microscope (Coleman $-1.25 \times$ magnification) (Giglioti et al., 2011).

\subsection{Statistical analysis}

For statistical analysis, the average of the quantification by sampling (performed in triplicate) of 210 syringes was calculated, since the technique of visual estimation was performed in only one observation, according to the original method of Drummond et al. (1973). Hatchability was categorized by scores $(1=0-25 \%, 2=26-50 \%$, $3=51-75 \%$, and $4=76-100 \%$ ) using as reference the quantification by sampling, since it is a quantitative method, performed in triplicate. Results were checked for normal distribution by PROC UNIVARIATE and for homogeneity of variances by Bartlett's test. Data were analyzed by the nonparametric model PROC NPAR1WAY. The averages of the scores between both techniques were compared by the Tukey test ( $p \leq$ 0.05). The general scores and per scale of both techniques were associated/correlated by the Pearson correlation coefficient. All statistical analyses were performed with the SAS package (SAS, 2002/2010SAS Institute, 2002SAS, 2002/2010).

\section{Results and discussion}

The analysis demonstrated the data were not normally distributed and the variances were heterogeneous, so a nonparametric model for analysis was used. The correlation analysis between the two techniques indicated high correlation (0.87), which was expected, since the same syringe was used for the two evaluations. The correlations between the two techniques separated by categories, based on the quantification by sampling, were significant $(\mathrm{p} \leq 0.05)$ and were as follows: category 1 : 0.74; 2: 0.38 ; 3: 0.60 and 4: 0.45 (Fig. 2). When associated by scales, there was a reduction in the value found in the general association between the two techniques. These findings show that the differences between the two techniques may be even greater, and can depend on the hatching rates of the larvae. The strongest correlation was found when the hatching rates were lower (category 1 ).

A statistically significant difference was found $(p<0.05)$ between the two tests, indicating that the average of the visual estimate (56.8) was higher than the average of the quantification by sampling (48.0). This demonstrates that in visual estimation, the number of hatched larvae can be overestimated, since the larvae tend to adhere to the wall and the infertile eggs can stay hidden in the center of the syringe. Such findings are in accordance with the discussion of Amaral (1993), who stated that visual assessment is subjective.

In relation to the quantification by sampling technique, statistical difference was not verified $(p=0.99)$ between the values obtained in the three-count sampling syringes. The correlation of repetitions was 0.96 , demonstrating that the number obtained by one count repetition is very close to the others (averages of $48.1 \pm 26.6 \%, 47.8 \pm 26.9 \%$, $48.1 \pm 26.5 \%)$. So, it can be assumed that only one count is necessary, provided the material is well homogenized. These analyses also suggest that the result of a syringe should not differ much if several evaluations are performed by different analysts. Some authors who have performed AIT by quantification have used other egg hatching evaluation techniques. In the study of Terassani et al. (2012), all larvae from 10 engorged females (in triplicate) submitted to plant extracts were killed in sulfuric ether and counted under a stereoscopic microscope. Vasconcelos et al. (2014) reported that all the syringe contents were counted under a stereoscopic microscope. On the other hand, Barbosa et al. (2013) reported that when less than $50 \%$ of the larvae hatched, eggs and larvae were mixed in $4 \mathrm{~mL}$ of a 1:1 solution of aqueous ethanol at $96 \%$ and glycerin. Then larvae and eggs were counted in $1 \mathrm{~mL}$ of the solution. In the study of Lopez-Arias et al. (2014), the egg mass of each tick was weighed, and then $12 \mathrm{mg}$ of eggs that were laid within the first 3-4 days from every tick were transferred into 5-mL sterile Vacutainer tubes. Hatchability was based on the number of larvae emerging from $12 \mathrm{mg}$ of eggs (it had been previously determined that 300 eggs weighed $12 \mathrm{mg}$ ). All larvae were removed from the tubes with distilled water, and then counted manually by immobilizing them with sticky tape. As 


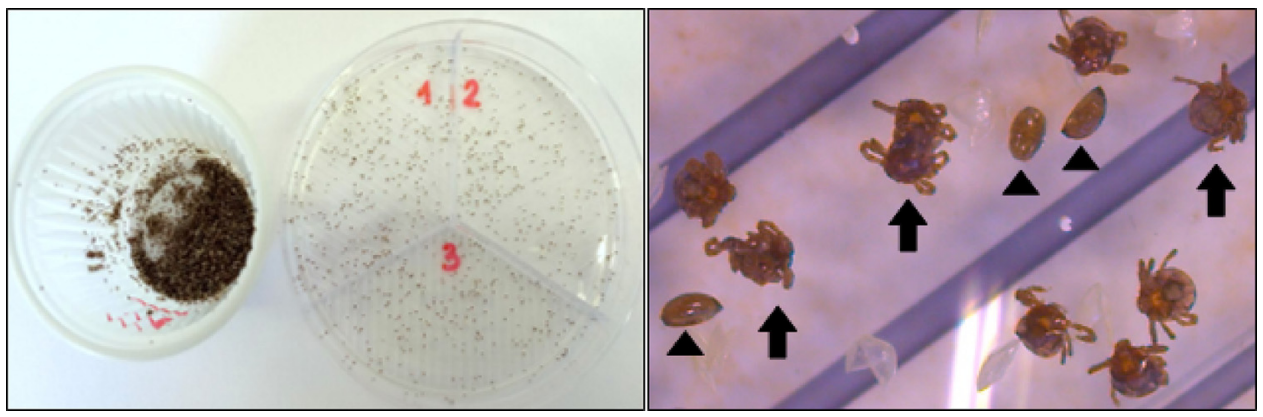

Fig. 1. The content of a syringe was homogenized and distributed in 3 areas of a disposable Petri dish $(90 \times 15 \mathrm{~cm})$ to count larvae and infertile eggs under a stereoscope (Coleman-1.25 magnification). Black arrow: larvae, black arrowheads: eggs.

can be noted, these researchers performed the quantification of all syringe contents or used some laborious process for larval counting. However, our results show that a single sample of 100 larvae/eggs is reliable.

The results found here can have a serious impact on the AIT performed to detect resistance status of tick populations, because when the efficacy of a commercial acaricide is $<95 \%$ by the AIT, this means tick resistance exists in the farm, requiring changing to a new chemical group. This evaluation criterion was established according to the guidelines of WAAVP (Holdsworth et al., 2006). However, when the
(A)

Category 1

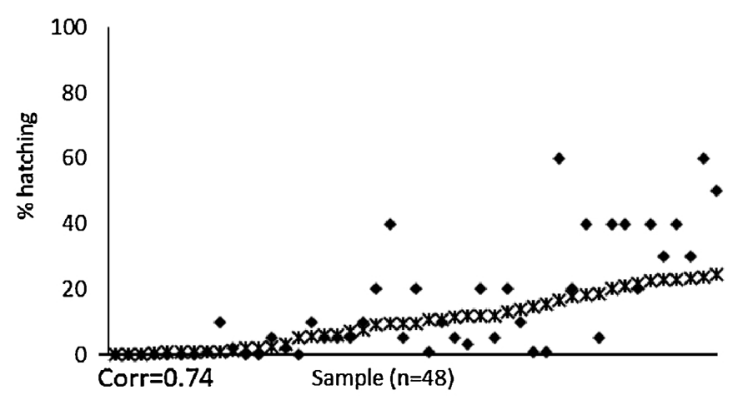

(C)

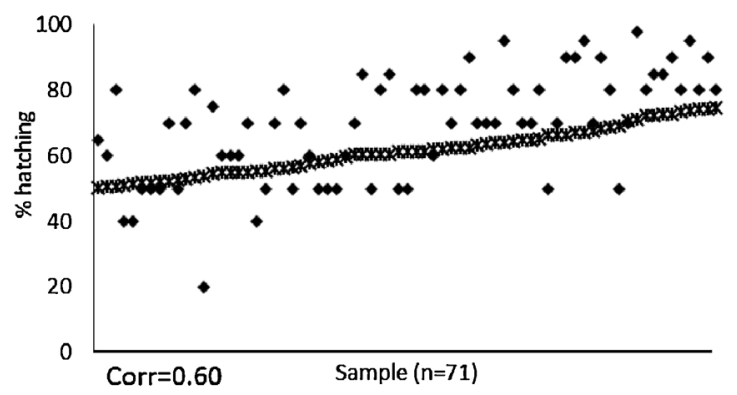

(B)

Category 2

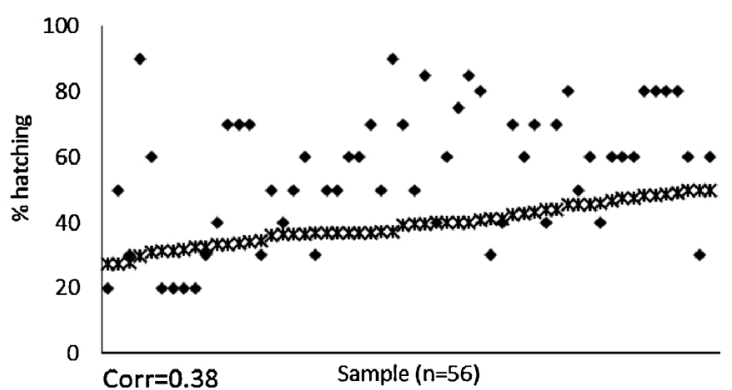

(D)

Category 4

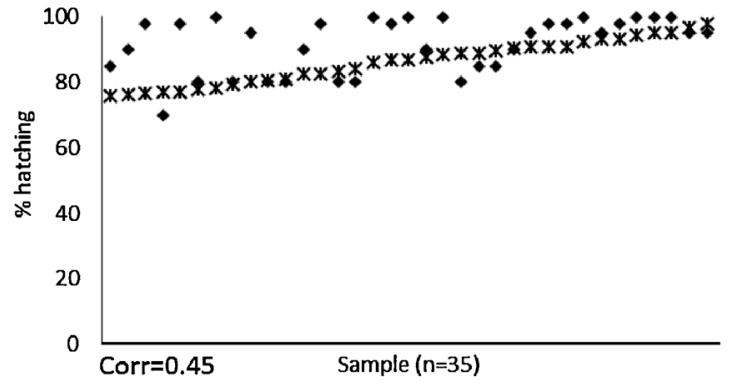

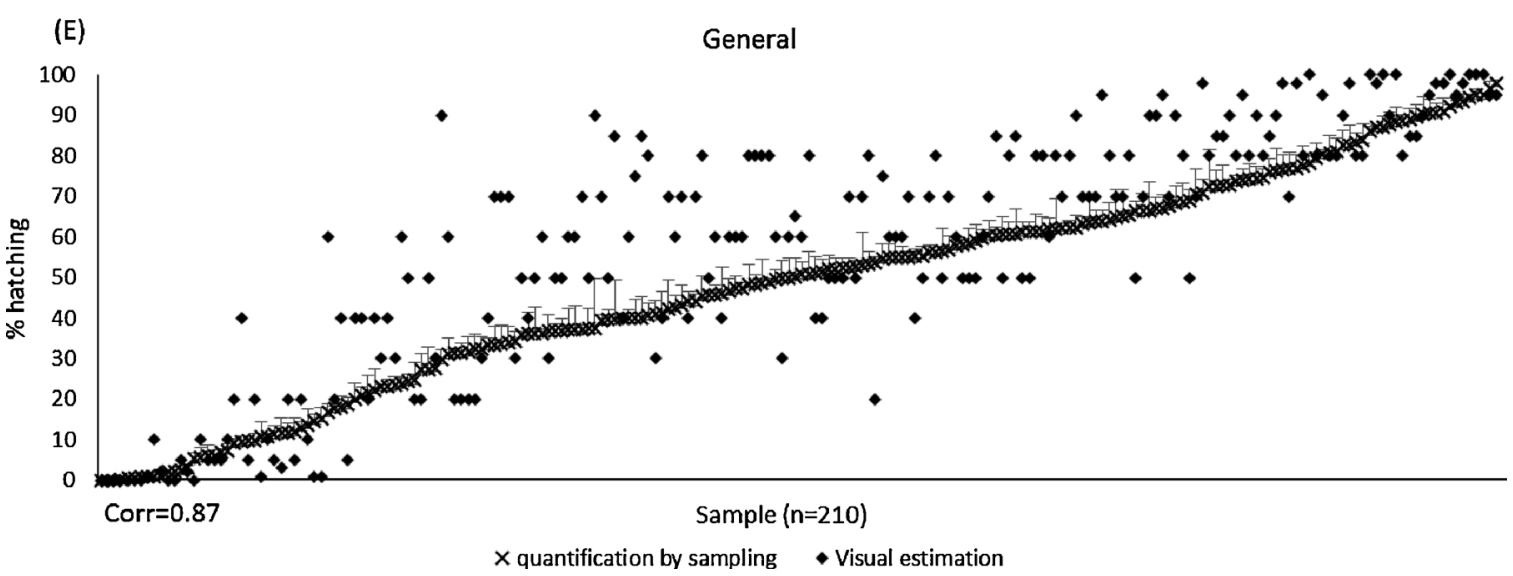

Fig. 2. Scattergram showing the correlation (Pearson $r$ ) of Rhipicephalus (Boophilus) microplus egg hatching between visual estimation and quantification by sampling, in each category (A: $1=0-25 \%$, B: $2=26-50 \%$, C: $3=51-75 \%$, D: $4=76-100 \%$ ) and in general (E: the standard deviation bars (grey bars) are related to the three counts in the quantification by sampling). 
Table 1

Averages of Rhipicephalus (Boophilus) microplus egg hatching evaluated by visual estimation and quantification by sampling, organized by categories.

\begin{tabular}{llll}
\hline Category $^{*}$ & Egg hatching & Visual estimation & Quantification by sampling \\
\hline 1 & $0-25 \%$ & $14.8 \pm 17.3 \mathrm{aA}$ & $10.7 \pm 8.2 \mathrm{aA}$ \\
2 & $26-50 \%$ & $55.4 \pm 20.0 \mathrm{bB}$ & $39.2 \pm 6.4 \mathrm{bA}$ \\
3 & $51-75 \%$ & $69.2 \pm 16.5 \mathrm{cB}$ & $61.4 \pm 7.1 \mathrm{cA}$ \\
4 & $76-100 \%$ & $91.6 \pm 8.5 \mathrm{dA}$ & $86.2 \pm 6.6 \mathrm{dA}$ \\
General & $0-100 \%$ & $56.8 \pm 30.6 \mathrm{~B}$ & $48.0 \pm 26.4 \mathrm{~A}$ \\
\hline
\end{tabular}

Different upper-case letters in the same line or lower-case in the same column indicate significant differences according to Tukey test $(p \leq 0.05)$.

* Categories defined based on the quantification by sampling.

results obtained in the quantification by sampling technique were split by category and compared, it was clear that in the extreme results, i.e., low hatching $(0-25 \%)$ or high hatching $(76-100 \%)$, there was no statistical difference (Table 1). So, at the cutoff point of tick resistance status (95\%), both methods are reliable, because it is necessary to have low egg hatching (probably $0-25 \%$ ) to reach a low estimated reproduction of the engorged females, and good efficacy of the acaricide. Statistical difference was seen in the intermediate categories (26-50\% and $51-75 \%$ ) and the overestimation of egg hatching by the visual estimation in both categories can have an impact on the calculation of the resistance status.

\section{Conclusions}

We can conclude that the quantification by sampling proved to be a more reliable technique in relation to visual estimation, which tends to overestimate the number of hatched larvae. At the extremes of egg hatching $(0-25 \%$ or $76-100 \%)$, there was no statistical difference. In turn, for the cutoff point of tick resistance status, both methods are reliable. Therefore, in studies that require greater scientific accuracy, the counting by sampling technique is recommended because it will lead to the same result from evaluating only one sample. We believe that this finding is of interest to other laboratories that perform the AIT routinely, because questions related to the reliability of visual estimation are relatively frequent, including by referees of peer reviewed journals.

\section{Competing interests}

The authors declare that they have no financial or personal relationships that may have inappropriately influenced them in writing this article.

\section{Authors' contributions}

AF designed this research. AF, ICA and LGL conducted experiments. ACSC contributed analytical tools. RG analyzed data. AF and ACSC wrote the manuscript. All authors read and approved the manuscript.

\section{Acknowledgments}

We thank the Conselho Nacional de Desenvolvimento Científico e
Tecnológico (CNPq) for the scholarships granted and Empresa Brasileira de Pesquisa Agropecuária (Embrapa) for funding this research (Project 02.13.01.001.00-00).

\section{References}

Amaral, N.K., 1993. Boophilus microplus (Canestrini, 1887) (Acari: Ixodidae). Rev. Bras. Parasitol. Vet. 2, 144-151.

Barbosa, C.S., Borges, L.M.F., Nicácio, J., Alves, R.D., Miguita, C.H., Violante, I.M.P., Hamerski, L., Garcez, W.S., Garcez, F.R., 2013. In vitro activities of plant extracts from the Brazilian Cerrado and Pantanal against Rhipicephalus (Boophilus) microplus (Acari: Ixodidae). Exp. Appl. Acarol. 60, 421-430.

Chagas, A.C.S., Oliveira, M.C.S., Giglioti, R., Santana, R.C.M., Bizzo, H.R., Gama, P.E., Chaves, F.C.M., 2016. Efficacy of 11 Brazilian essential oils on lethality of the cattle tick Rhipicephalus (Boophilus) microplus. Ticks Tick Borne Dis. 7, 427-432.

Drummond, R.O., Ernest, S.E., Trevino, J.L., Gladney, W.J., Graham, O.H., 1973. Boophilus annulatus and Boophilus microplus: laboratory tests for insecticides. J. Econ. Entomol. 66, 130-133.

Gaur, R.S., Sangwan, A.K., Sangwan, N., Kumar, S., 2016. Acaricide resistance in Rhipicephalus (Boophilus) microplus and Hyalomma anatolicum collected from Haryana and Rajasthan states of India. Exp. Appl. Acarol. 69, 487-500.

Giglioti, R., Forim, M.R., Oliveira, H.N., Chagas, A.C.S., Ferrezini, J., Brito, L.G., Falcoski, T.O.R.S., Albuquerque, L.G., Oliveira, M.C.S., 2011. In vitro acaricidal activity of neem (Azadirachta indica) seed extracts with known azadirachtin concentrations against Rhipicephalus microplus. Vet. Parasitol. 181, 309-315.

Grisi, L., Leite, R.C., Martins, J.R.S., Barros, A.T.M., Andreotti, R., Cançado, P.H.D., Perez de León, A.A., Pereira, J.B., Villela, H.S., 2014. Reassessment of the potential economic impact of cattle parasites in Brazil. Braz. J. Vet. Parasitol. 23, 150-156.

Habeeb, S.M., 2010. Ethno-veterinary and medical knowledge of crude plant extracts and its methods of application (traditional and modern) for tick control. World Appl. Sci. J. 9, 1047-1054.

Holdsworth, P.A., Kemp, D., Green, P., Peter, R.J., De Bruin, C., Jonsson, N.N., Letonja, N.N., Rehbein, S., Vercruysse, J., 2006. World Association for the Advancement of Veterinary Parasitology (W. A. A. V. P.) guidelines for evaluating the efficacy of acaricides against ticks (Ixodidae) on ruminants. Vet. Parasitol. 136, 29-43.

Klafke, G.M., Castro-Janer, E., Mendes, M.C., Namindome, A., Schumaker, T.T., 2012. Applicability of in vitro bioassays for the diagnosis of ivermectin resistance in Rhipicephalus microplus (Acari: Ixodidae). Vet. Parasitol. 184, 212-220.

Lopez-Arias, A., Villar-Argaiz, D., Chaparro-Gutierrez, J.J., Miller, R.J., Leon, A.A.P., 2014. Reduced efficacy of commercial acaricides against populations of resistant cattle tick Rhipicephalus microplus from two municipalities of Antioquia, Colombia. Environ. Health Perspect. 8, 71-80.

Raynal, J.T., Silva, A.A.B., Sousa, T.J., Bahiense, T.C., Meyer, R., Portela, R.W., 2013. Acaricides efficiency on Rhipicephalus (Boophilus) microplus from Bahia state NorthCentral region. Rev. Bras. Parasitol. 22, 71-77.

Reck, J., Klafke, G.M., Webster, A., Dall'Agnol, B., Scheffer, R., Souza, A.U., Coraqssini, B.V., Vargas, R., dos Santos, S.J., Martins, J.R., 2014. First report of fluazuron resistance in Rhipicephalus microplus: a field tick population resistant to six classes of acaricides. Vet. Parasitol. 201, 128-136.

SAS Institute, 2002/2010. SAS/INSIGHT User's Guide, 9.1.3 Version, Windows Version. Cary, NC, USA.

Schwarzenbach, R.P., Egli, P., Hofstetter, T.B., Gunten, U., Wehrli, B., 2010. Global water pollution and human health. Annu. Rev. Environ. Resour. 35, 109-136.

Terassani, E., Santos, H.J., Silva, I.D., Cardoso, B.K., Souza, S.G.H., Gazim, Z.C., 2012 Efeito do extrato de Azadirachta indica em carrapatos (Rhipicephalus (Boophilus) microplus). Arq. Ciênc. Vet. Zool. 15, 197-200.

Vasconcelos, V.O., Martins, M.A.D., Oliveira, N.J.F., Duarte, E.R., 2014. Effect of ethanolic extract of Capsicum frutescens L. on adult female of Rhipicephalus microplus (Ixodidae). Parasitol. Res. 113, 1389-1394.

Von Samson-Himmelstjerna, G., Coles, G.C., Jackson, F., Bauer, C., Borgsteede, F., Cirak, V.Y., Demeler, J., Donnan, A., Dorny, P., Epe, C., Harder, A., Hoglund, J., Kaminsky, R., Kerboeuf, D., Kuttler, U., Papadopoulos, E., Posedi, J., Small, J., Varady, M., Vercruysse, J., Wirtherle, N., 2009. Standardization of the egg hatch test for the detection of benzimidazole resistance in parasitic nematodes. Parasitol. Res. 105, 825-834. 